\title{
Uso do hábitat, atividade e comportamento de Bothriopsis bilineatus e de Bothrops atrox (Serpentes: Viperidae) na floresta do Rio Moa, Acre, Brasil
}

\author{
Luiz Carlos Batista Turci', Saymon de Albuquerque ${ }^{1}$, \\ Paulo Sérgio Bernarde ${ }^{2,3}$ \& Daniele Bazzo Miranda ${ }^{1}$ \\ ${ }^{1}$ Programa de Pós-Graduação em Ecologia e Manejo de Recursos Naturais, \\ Universidade Federal do Acre - UFAC, \\ Rod. BR-363, Km 04, Distrito Industrial, CEP 69915-900, Rio Branco, AC, Brasil \\ ${ }^{2}$ Laboratório de Herpetologia, Centro Multidisciplinar, Campus Floresta, \\ Universidade Federal do Acre - UFAC \\ CEP 69980-000, Cruzeiro do Sul, AC, Brasil \\ ${ }^{3}$ Autor para correspondência: Paulo Sérgio Bernarde, email: snakebernarde@hotmail.com
}

TURCI, L. C. B., ALBUQUERQUE, S., BERNARDE, P. S. \& MIRANDA, D. B. Activity, habitat use, and behavior of the Bothriopsis bilineatus and of the Bothrops atrox (Serpentes: Viperidae) in Moa river forest, Acre Brazil. Biota Neotrop., 9(3): http://www.biotaneotropica.org.br/v9n3/en/abstract?article+bn03909032009.

\begin{abstract}
The snakes Bothriopsis bilineatus and Bothrops atrox are sympatric vipers in the Amazon. While B. atrox is common, Bothriopsis bilineatus is considered relatively rare. Both species are dietary generalists, with $B$. atrox foraging mostly on the ground while $B$. bilineatus is more arboreal. Here, we describe habitat use and activity patterns for these two snakes in Amazonian forest near the Moa River, Cruzeiro do Sul (Acre). This study was based on observations during both time constrained visual searches and opportunistic encounters along trails that included both seasonally flooded and terra firme forests. During a total of 360 hours of time constrained visual search, 11 B. bilineatus and six $B$. atrox were found, while one B. bilineatus and six B. atrox were found during opportunistic encounters. All 12 B. bilineatus were found on vegetation above the ground, while only five $B$. atrox were on vegetation, and these were all juveniles. Thus, during time constrained visual searches $B$. bilineatus was more common ( 0.03 snake/hour) than B. atrox (0.016 snake/hour). These two species use different habitats. Bothriopsis bilineatus was most commonly found in upland terra firme forests, with many palms and free from seasonal floods. Bothrops atrox was most commonly found in lower, wetter seasonally flooded areas. Perhaps structural differences in the vegetation in these two forest types influence the distribution of these two species, possibly due to a related influence on prey abundance, besides historical factors. Both species are found more often during the rainy season, rarely being found during the dry season, when their amphibian prey are less abundant. In most of the Amazon, B. bilineatus seems less common than B. atrox, but in the present study, the former was the most frequent snake during time constrained visual search.
\end{abstract}

Keywords: Western Amazonia, Reptilia, pitvipers, lanceheads, microhabitat, snake behavior.

TURCI, L. C. B., AlBuQUerque, S., BERnARDE, P. S. \& MIRANDA, D. B. Uso do hábitat, atividade e comportamento de Bothriopsis bilineatus e de Bothrops atrox (Serpentes: Viperidae) na floresta do Rio Moa, Acre - Brasil. Biota Neotrop., 9(3): http://www.biotaneotropica.org.br/v9n3/pt/ abstract?article+bn03909032009.

Resumo: As serpentes Bothriopsis bilineatus e Bothrops atrox são viperídeos simpátricos na Amazônia, sendo B. atrox comum e $B$. bilineatus mais rara. Ambas espécies apresentam uma dieta generalista, sendo que $B$. atrox forrageia principalmente sobre o chão e $B$. bilineatus é mais arborícola. Aqui, nós descrevemos o uso do habitat e padrão de atividade dessas duas serpentes em uma floresta amazônica no Rio Moa, Cruzeiro do Sul, Acre. Esse estudo foi baseado em observações durante procura visual limitada por tempo e encontros ocasionais de serpentes em uma trilha que é sazonalmente alagada e outra de floresta de terra firme. Durante um total de 360 horas de procura visual limitada por tempo, 11 B. bilineatus e seis $B$. atrox foram encontradas, enquanto uma B. bilineatus e seis $B$. atrox foram encontradas durante encontros ocasionais. Todas 12 B. bilineatus foram observadas sobre a vegetação, enquanto somente cinco $B$. atrox estavam sobre a vegetação (todos juvenis). Durante procura visual limitada por tempo $B$. bilineatus foi mais comum ( 0,03 serpentes/hora) do que $B$. atrox ( 0,016 serpentes/hora). Essas duas espécies apresentaram diferenças no uso do espaço, sendo que $B$. bilineatus ocorreu principalmente na área da floresta de terra firme, rica em palmeiras e que não é sazonalmente alagada. Bothrops atrox foi mais abundante na área da floresta próxima aos lagos, que é sazonalmente alagada. Diferenças entre essas duas áreas como a estrutura da vegetação, tipos de ambientes aquáticos, disponibilidade de presas e fatores históricos são possivelmente os fatores responsáveis pela diferença na ocorrência dessas espécies. Estas duas serpentes foram encontradas nos meses mais chuvosos e não foram registradas nos meses mais secos, período em que também existe menor disponibilidade de anuros nesses ambientes. Na maior parte da Amazônia, B. bilineatus parece ser menos frequente que B. atrox, mas na área do presente estudo, a primeira parece ser mais frequente que a última durante procura visual limitada por tempo.

Palavras-chave: Amazônia ocidental, Reptilia, jararacas, microhabitat, comportamento de serpentes. 


\section{Introdução}

A Amazônia brasileira apresenta uma rica fauna de serpentes com aproximadamente 149 espécies registradas (Ávila-Pires et al. 2007). Pouco se conhece sobre a ecologia e história natural da maioria dessas espécies. A detectibilidade de serpentes na natureza é relativamente mais difícil do que o de outros grupos de vertebrados e alguns métodos de amostragem, como a procura visual limitada por tempo e armadilhas de interceptação e queda, são os métodos mais usuais para amostrar estes animais (e. g., Martins \& Oliveira 1998, Cechin \& Martins 2000, Bernarde \& Abe 2006). A procura limitada por tempo permite que sejam coligidas informações precisas sobre a atividade do animal e também possibilita a comparação da taxa de encontros de serpentes entre diferentes ambientes ou mesmo com outros estudos (Martins \& Oliveira 1998, Oliveira \& Martins 2001).

Alguns estudos sobre viperídeos utilizando a procura visual contribuíram de forma significativa para o conhecimento da ecologia e história natural de algumas espécies no Brasil (e. g. Sazima 1988, Oliveira \& Martins 2001, Valdujo et al. 2002, Hartmann et al. 2003, Nogueira et al. 2003, Hartmann et al. 2005). Esses estudos revelaram informações sobre uso de ambientes, uso do microhábitat de atividade e de repouso, além de alguns aspectos sobre a história natural das espécies, como o comportamento alimentar.

Os viperídeos Bothriopsis bilineatus (Wied 1825) e Bothrops atrox (Linnaeus 1758) ocorrem na Amazônia, sendo que existem mais estudos com a segunda espécie, provavelmente por ser uma das mais abundantes nessa região (Martins \& Oliveira 1998, Oliveira \& Martins 2001). Bothriopsis bilineatus apresenta atividade noturna, hábitos arborícolas e é uma serpente pouco frequiente nos estudos herpetofaunísticos (Duellman 1978, Dixon \& Soini 1986, Cunha \& Nascimento 1993, Jorge-da-Silva 1993, Martins et al. 2001, Bernarde \& Abe 2006). Alimenta-se principalmente de roedores e anuros, e também de serpentes e lagartos (Dixon \& Soini 1986, Cunha \& Nascimento 1993, Martins et al. 2001). Dixon \& Soini (1986) também mencionaram pequenos pássaros encontrados no conteúdo estomacal dessa espécie. Bothrops atrox apresenta atividade predominantemente noturna; o adulto caça preferencialmente no chão, enquanto os juvenis são mais encontrados sobre a vegetação (Martins \& Oliveira 1998, Oliveira \& Martins 2001). Essa espécie apresenta uma dieta generalista, predando centopéias, peixes, anuros, lagartos, outras serpentes, aves e pequenos mamíferos (Martins \& Gordo 1993, Egler et al. 1996, Martins \& Oliveira 1998, Martins et al. 2002, Oliveira \& Martins 2003, Macedo-Bernarde \& Bernarde 2005, Nascimento et al. 2008). Estas duas espécies pertencem a linhagens diferentes, sendo o clado de Bothrops composto por espécies terrestres e subarborícolas e com dieta generalista e Bothriopsis espécies subarborícolas com dieta generalista (Fenwick et al. 2009).

Com o objetivo de contribuir para o conhecimento da história natural e ecologia das serpentes amazônicas, são apresentados nesse estudo dados sobre uso do hábitat e atividade de Bothriopsis bilineatus e Bothrops atrox na Floresta do Baixo Rio Moa, Cruzeiro do Sul (Acre), através de registros feitos durante procura visual limitada por tempo e por encontros ocasionais.

\section{Material e Métodos}

\section{1. Área de estudo}

A floresta do baixo Rio Moa (07 37' 14,7' S e 72 48’ 09,9”'W) se enquadra na formação vegetacional "Floresta Aluvial Aberta com Palmeira” (ZEE 2006), e localiza-se no município de Cruzeiro do Sul, no Estado do Acre. O Rio Moa é afluente do Rio Juruá, no extremo oeste do Brasil. O clima da região é caracterizado como tropical, quente, úmido, com temperatura média anual de $24^{\circ} \mathrm{C}$ (Ribeiro 1977), sendo período entre os meses de maio a outubro a parte mais seca do ano. Durante o período deste estudo (abril de 2008 a março de 2009) apresentou uma precipitação pluviométrica anual de $2405 \mathrm{~mm}$. Os dados climáticos foram obtidos na Estação Meteorológica de Cruzeiro do Sul do Instituto Nacional de Meteorologia (INMET). O estudo foi desenvolvido em duas trilhas (com $2 \mathrm{~km}$ cada) localizadas em diferentes tipos de ambientes, com uma distância média de aproximadamente $1,5 \mathrm{~km}$ entre elas:

Trilha dos lagos (Figuras 1a, 2 e 3): Floresta Aluvial Densa com árvores emergentes (ZEE 2006). Essa tipologia florestal ocorre ao longo dos principais rios e alguns dos seus afluentes (ZEE 2006) e, devido sua maior proximidade ao Rio Moa, sofre influência deste, sendo sazonalmente alagada com o enchimento dos lagos. O dossel florestal apresenta árvores com uma altura média em torno de $20 \mathrm{~m}$ e nessa área também ocorrem algumas espécies emergentes atingindo altura máxima de $35 \mathrm{~m}$. O dossel é fechado, sendo composto principalmente por indivíduos do gênero Brosimum (Moraceae), Enterolobium (Fabaceae) e Virola (Myristicaceae). Grande parte das árvores de grande porte apresenta raízes tabulares (sapopembas), comuns em espécies que ocorrem em solos instáveis e que sofrem influência das cheias durante um período do ano. O sub-bosque é caracterizado pela grande abundância de Poaceae, Astrocaryum (Arecaceae) e arvoretas e pela pouca abundância de ervas (Heliconiaceae, Marantaceae e Costaceae). A vegetação apresentou-se pouco abundante para repre-
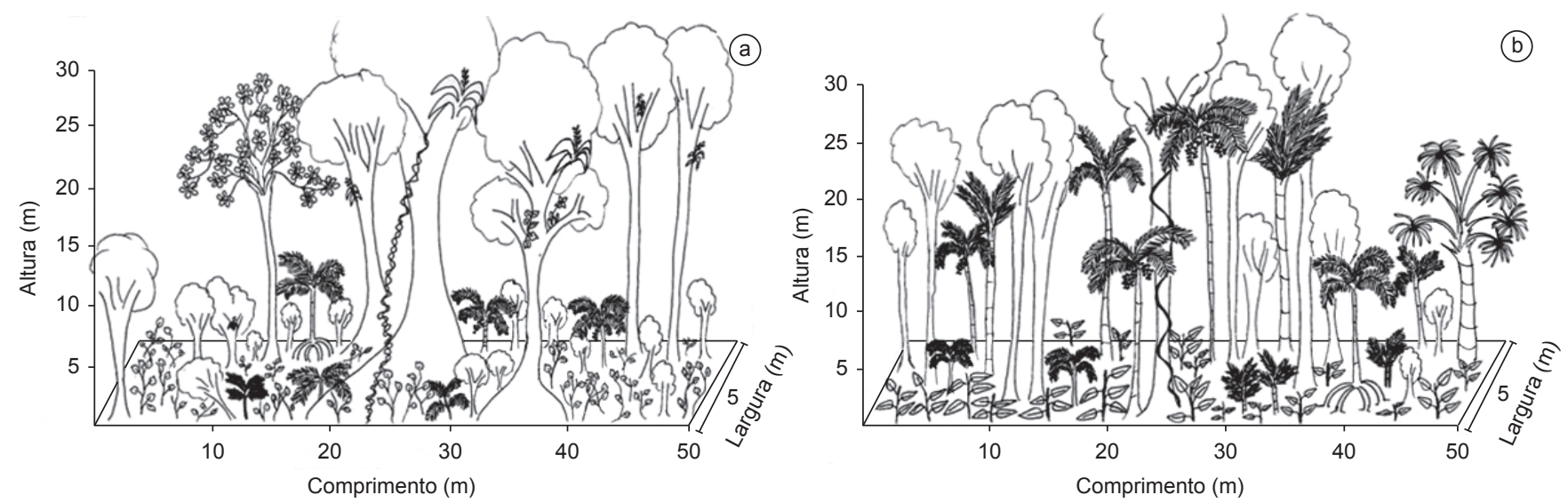

Figura 1. Desenhos representando a estrutura da vegetação das trilhas dos lagos (a) e de terra firme (b) na floresta do Rio Moa (AC).

Figure 1. Drawings representing the structure vegetation of lakes (a) and terra firme (b) trails at Moa river forest (AC). 


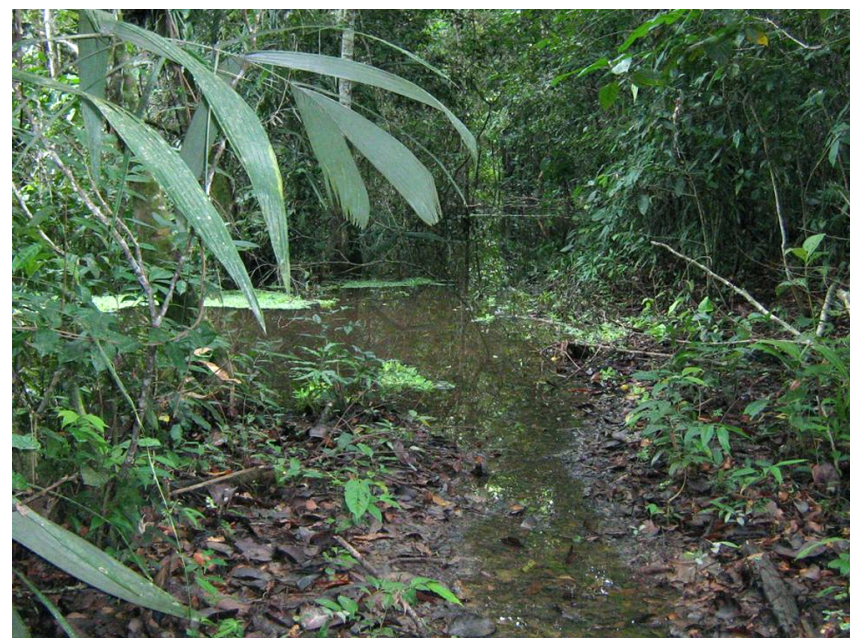

Figura 2. Trilha dos lagos na floresta do Rio Moa (AC).

Figure 2. Lagos trail at Moa river forest (AC).

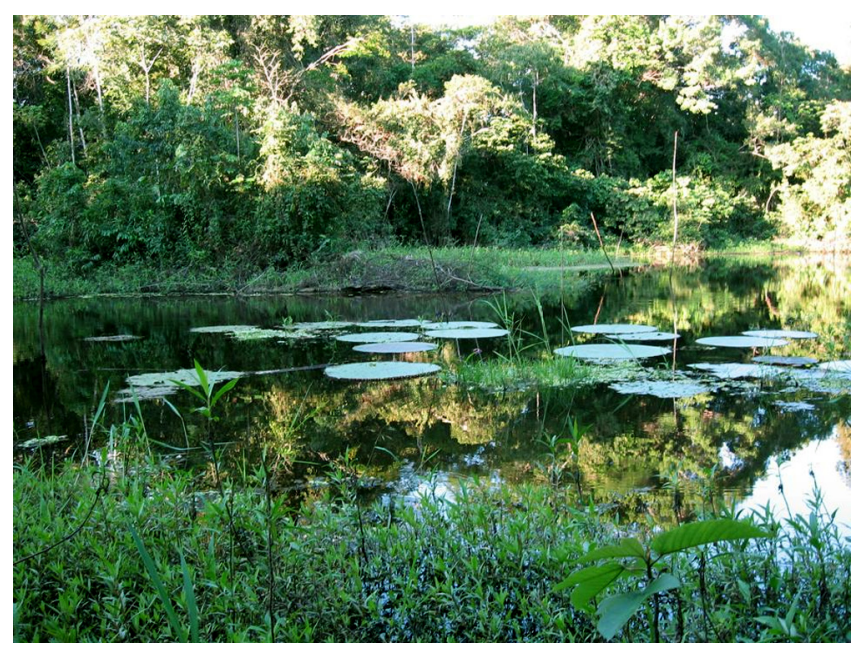

Figura 3. Lago na floresta do Rio Moa (AC).

Figure 3. Lake at Moa river forest (AC).

sentantes da família Arecaceae (Palmeiras), sendo observados alguns indivíduos das espécies Euterpe precatória, Iriartella stenocarpa e Bactris sp. Em toda sua extensão essa trilha margeia três lagos que invadem a trilha durante a cheia (janeiro a março) por influência do Rio Moa.

Trilha em terra firme (Figuras 1b, 4 e 5): Floresta Aberta com Palmeiras de Terra Firme (ZEE 2006). Essa tipologia vegetal é geralmente encontrada em áreas próximas a planícies aluviais de rios com grande vazão na época da cheia e se caracteriza por uma floresta de dossel aberto com presença de palmeiras, podendo também ser encontradas áreas com cipós (ZEE, 2006). As árvores que atingem o dossel e que apresentam os maiores diâmetros são representadas principalmente pelos gêneros Couma (Apocynaceae), Parkia e Vataireopsis (Fabaceae), Virola (Myristicaceae), Pouteria e Manilkara (Sapotaceae) e Vochysiaceae, não sendo comuns nessas espécies a presença de raízes tabulares. A altura média do dossel foi de aproximadamente $20 \mathrm{~m}$, com uma altura máxima de $30 \mathrm{~m}$, sendo aberto e comum a presença de palmeiras (Arecaceae): Astrocaryum jauari, Mauritia flexuosa, Euterpe precatoria, Iriartea deltoidea e Iriartella stenocarpa. Abaixo do dossel, na região de sub-bosque, a vegetação apresenta-se com poucos arbustos e a estratificação da floresta é pouco definida, o que é

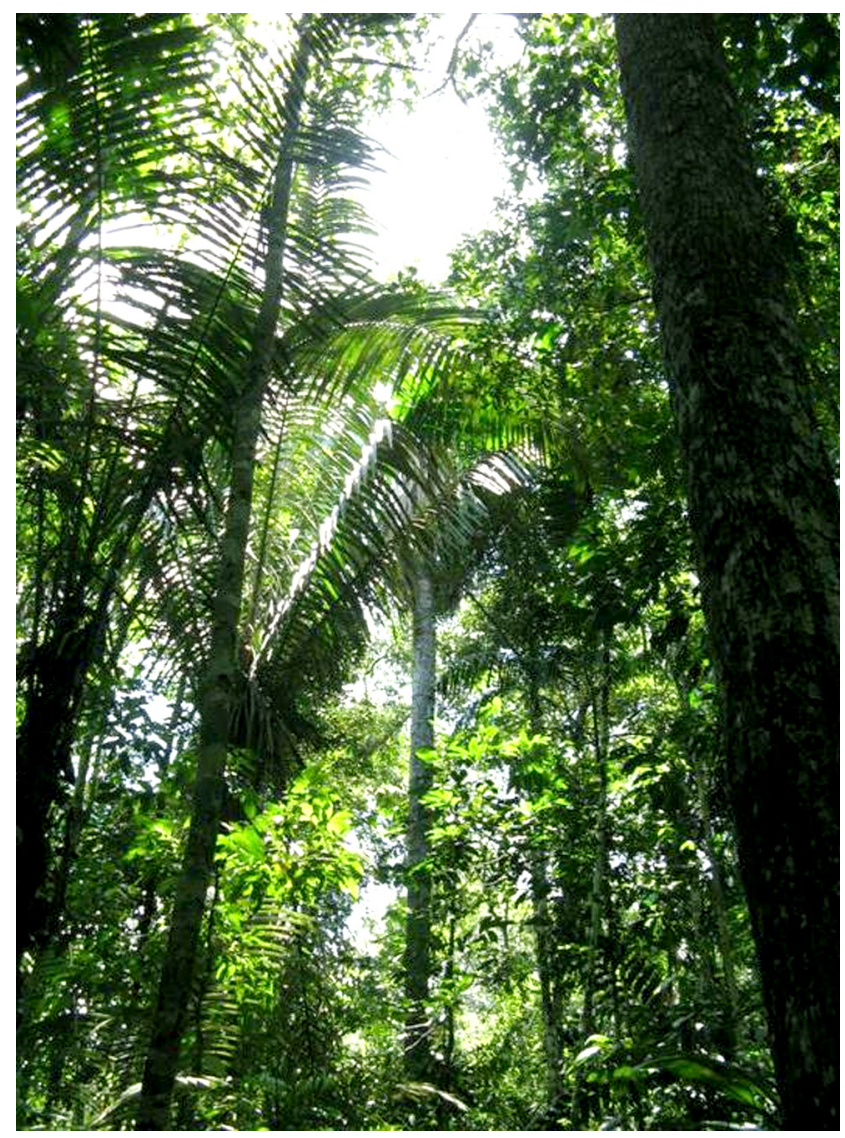

Figura 4. Trilha em terra firme na floresta do Rio Moa (AC).

Figure 4. Terra firme trail at Moa river forest (AC).

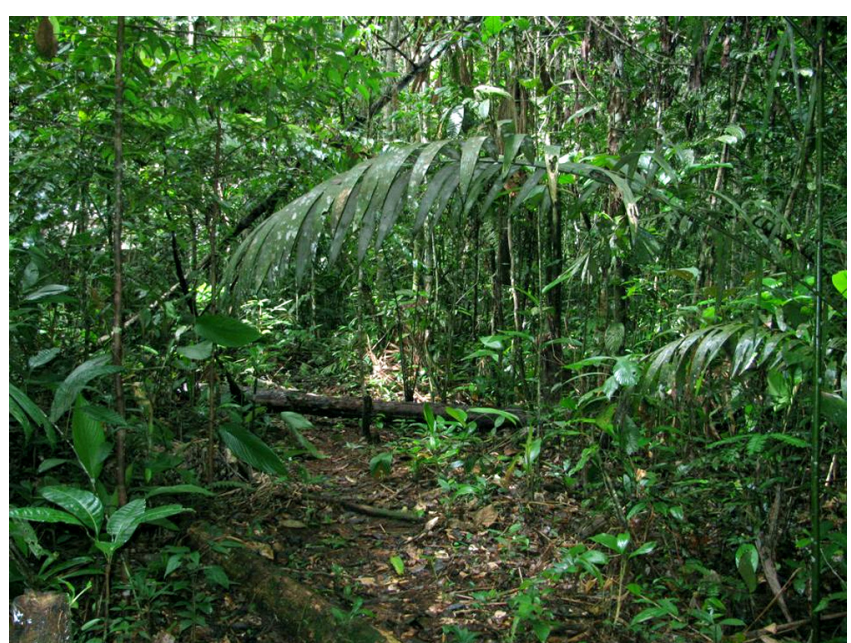

Figura 5. Trilha em terra firme na floresta do Rio Moa (AC).

Figure 5. Terra firme trail at Moa river forest (AC).

comum em florestas abertas devido à maior entrada de luz sobre a região de sub-bosque (ZEE 2006). Nesse ambiente a vegetação se mostrou bastante densa, sendo comum a presença de Bactris sp. (Arecaceae), e em maior abundância ervas representadas pela espécie Calathea sp. (Marantaceae), não sendo rara a ocorrência das famílias Heliconiaceae e Cyperaceae. Quanto à presença de ambientes aquáticos, existe um riacho temporário (cerca de $3 \mathrm{~m}$ de largura) que seca sazonalmente e é margeado pela trilha ao longo de sua extensão. 


\section{Métodos de amostragem}

Procura visual limitada por tempo (PVLT) (ver Campbell \& Christman 1982, Martins \& Oliveira 1998): Consistiu no deslocamento a pé, lentamente através das trilhas dentro de floresta, à procura de serpentes que estavam visualmente expostas. Foram realizadas 360 horas de procura (288 horas durante a noite e 72 horas durante o dia, pela manhã) nas duas trilhas durante 12 meses (abril de 2008 - março de 2009). Em cada mês, foram realizadas 12 horas de procura noturna em cada trilha, percorrendo cerca de 800 metros; e três horas de procura diurna percorrendo aproximadamente 200 metros. Para a quantificação do esforço amostral, foi utilizada a taxa de encontro baseada no tempo de procura, ou seja, o número de indivíduos encontrados por hora-observador de procura.

Foram também registrados os indivíduos encontrados nessas duas trilhas fora do período de procura visual limitada por tempo. Para cada serpente encontrada foi anotado o horário, altura do substrato e sua atividade no momento do encontro (caçando, repousando, deslocando-se). Os indivíduos observados foram fotografados e alguns capturados para determinação do sexo e para a obtenção de medidas. As medidas apresentadas das serpentes são comprimento rostro-anal (CRA), comprimento da cauda (CC) e o peso (P), sendo respectivamente em centímetros $(\mathrm{cm})$ e gramas $(\mathrm{g})$. Dois indivíduos de Bothriopsis bilineatus e quatro de Bothrops atrox foram coletados para material testemunho e depositados na Coleção Herpetológica da Universidade Federal do Acre, Campus Floresta, em Cruzeiro do Sul (B. bilineatus UFACF 982, 1281; B. atrox UFACF 1097, 1098, $1099,1175)$. A atividade foi inferida a partir da postura que a serpente se encontrava (Oliveira \& Martins 2001), sendo considerada ativa quando se encontrava exposta, em deslocamento ou enrodilhada com o pescoço em "S" e a cabeça sobre volta do corpo, geralmente formando um ângulo maior ou igual a $20^{\circ}$ em relação ao substrato. Foram consideradas inativas (em repouso) as serpentes que foram encontradas geralmente escondidas, frouxamente enrodilhadas, com a cabeça formando um ângulo menor que $20^{\circ}$ em relação ao substrato (Oliveira \& Martins 2001). Para o registro das interações entre três indivíduos de $B$. bilineatus foi adotado o método de observação de todas as ocorrências ("all ocurrences sampling") e amostragem de sequências ("sequence sampling") (Lehner 1996).

\section{Resultados}

Durante o período de um ano foram realizados 12 registros de Bothriopsis bilineatus e outros 12 de Bothrops atrox (Tabela 1). Onze registros de $B$. bilineatus e seis de $B$. atrox foram realizados durante PVLT, enquanto um de B. bilineatus e outros sete de $B$. atrox, por encontros ocasionais. A maioria dos encontros $(\mathrm{n}=10)$ de $B$. bilineatus ocorreu na trilha em terra firme e de $B$. atrox $(\mathrm{n}=10)$ na trilha dos

Tabela 1. Ocorrência de indivíduos de Bothriopsis bilineatus e Bothrops atrox na floresta do Rio Moa (AC). J = juvenis, Veg. = vegetação, EO = encontros ocasionais, PVLT = procura visual limitada por tempo.

Table 1. Occurrence of individuals of Bothriopsis bilineatus and Bothrops atrox at Moa river forest (AC). $\mathrm{J}=$ juveniles, Veg. $=$ vegetation, EO = opportunistic sightings, PVLT = time constrained visual search.

\begin{tabular}{|c|c|c|c|c|}
\hline Espécime & Data e horário & Método & Substrato & Atividade \\
\hline B. bilineatus $\widehat{\widehat{O}}$ & $24 / 09 / 0820: 00 \mathrm{~h}$ & PVLT & Veg. $180 \mathrm{~cm}$ & Caçando de espera \\
\hline B. bilineatus & 19/10/08 19:20 h & PVLT & Veg. $250 \mathrm{~cm}$ & Caçando de espera \\
\hline B. bilineatus J & $19 / 10 / 0820: 50 \mathrm{~h}$ & PVLT & Veg. $700 \mathrm{~cm}$ & Caçando de espera \\
\hline B. bilineatus * & 20/10/08 09:10 h & PVLT & Veg. $170 \mathrm{~cm}$ & Repouso \\
\hline B. bilineatus $\mathrm{J} *$ & 20/10/08 09:40 h & PVLT & Veg. $300 \mathrm{~cm}$ & Repouso \\
\hline B. bilineatus J & 20/10/08 08:40 h & PVLT & Veg. $50 \mathrm{~cm}$ & Repouso \\
\hline B. bilineatus & $12 / 11 / 0821: 19 \mathrm{~h}$ & PVLT & Veg. $250 \mathrm{~cm}$ & Deslocando-se \\
\hline B. bilineatus ㅇ & 27/01/09 19:15 h & PVLT & Veg. $30 \mathrm{~cm}$ & Caçando de espera \\
\hline B. bilineatus $9 *$ & 28/01/09 20:20 h & PVLT & Veg. $60 \mathrm{~cm}$ & Repouso \\
\hline B. bilineatus $\widehat{\widehat{O}}$ & 28/01/09 20:20 h & PVLT & Veg. $60 \mathrm{~cm}$ & Tentativa de corte \\
\hline B. bilineatus $\hat{\text { रे }}$ & 28/01/09 20:20 h & PVLT & Veg. $500 \mathrm{~cm}$ & Deslocando-se \\
\hline B. bilineatus + & $31 / 01 / 0910: 00 \mathrm{~h}$ & PVLT & Veg. $60 \mathrm{~cm}$ & Repouso \\
\hline B. atrox $q$ & 28/09/08 20:00 h & EO & Chão & Caçando de espera \\
\hline B. atrox $\odot \mathrm{J}$ & 17/10/08 19:00 h & PVLT & Veg. $40 \mathrm{~cm}$ & Deslocando-se \\
\hline B. atrox $\widehat{\jmath} \mathrm{J}$ & 17/10/08 19:43 h & PVLT & Veg. $150 \mathrm{~cm}$ & Deslocando-se \\
\hline B. atrox $甲 \mathbf{J}$ & $17 / 10 / 0821: 30 \mathrm{~h}$ & PVLT & Veg. $50 \mathrm{~cm}$ & Caçando de espera \\
\hline B. atrox $q \mathrm{~J}$ & 04/12/08 14:00 h & $\mathrm{EO}$ & Chão & Repouso \\
\hline B. atrox $\mathrm{J}$ & 26/01/09 09:00 h & $\mathrm{EO}$ & Chão & Encontrado morto \\
\hline B. atrox $\mathrm{J}$ & $30 / 01 / 0920: 10 \mathrm{~h}$ & PVLT & Veg. $40 \mathrm{~cm}$ & Caçando de espera \\
\hline B. atrox $\mathrm{J}$ & 20/02/09 18:30 h & EO & Chão & Deslocando-se \\
\hline B. atrox $\mathrm{J}$ & 20/02/09 20:00 h & PVLT & Veg. $30 \mathrm{~cm}$ & Caçando de espera \\
\hline B. atrox & 20/02/09 22:10 h & EO & Chão & Deslocando-se \\
\hline B. atrox & 27/02/09 09:00 h & $\mathrm{EO}$ & Chão & Repouso \\
\hline B. atrox & 26/03/09 19:40 h & PVLT & Chão & Caçando de espera \\
\hline
\end{tabular}

* Reencontro de indivíduo observado em noite anterior. 
Tabela 2. Taxa de encontro de Bothriopsis bilineatus $(\mathrm{n}=11)$ e Bothrops atrox $(\mathrm{n}=6)$ durante procura visual limitada por tempo na Floresta do Rio Moa (AC) entre abril de 2008 a março de 2009.

Table 2. Encounter rate of Bothriopsis bilineatus $(\mathrm{n}=11)$ and Bothrops atrox $(\mathrm{n}=6)$ during time constrained visual search at Moa river forest $(\mathrm{AC})$ from April 2008 to March 2009.

\begin{tabular}{lll}
\hline & \multicolumn{1}{c}{ B. bilineatus } & \multicolumn{1}{c}{ B. atrox } \\
\hline PVLT total (360 horas) & 0,03 serpentes/h ou 1 a cada 32 horas & 0,016 serpente/h ou 1 a cada 60 horas \\
PVLT noturna (288 horas) & 0,024 serpente/h ou 1 a cada 41 horas & 0,020 serpente/h ou 1 a cada 48 horas \\
PVLT diurna (72 horas) & 0,05 serpente/h ou 1 a cada 18 horas & 0 \\
\hline
\end{tabular}

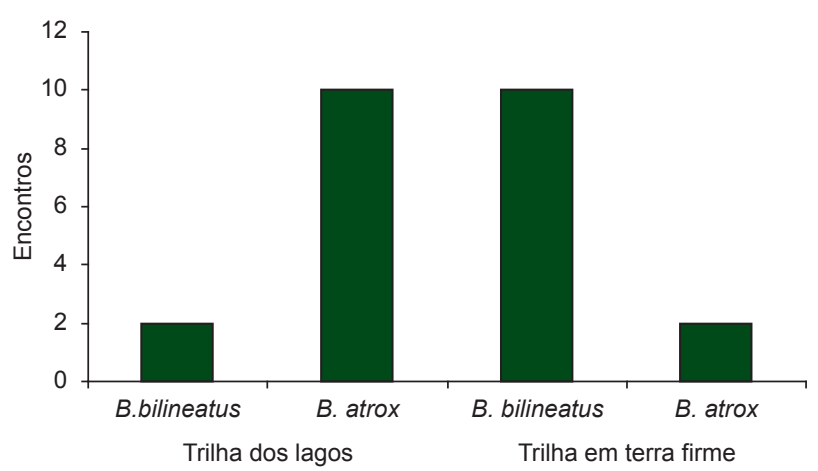

Figura 6. Ocorrência de Bothriopsis bilineatus $(\mathrm{n}=12)$ e de Bothrops atrox $(\mathrm{n}=12)$ na trilha dos lagos e na trilha em terra firme na Floresta do Rio Moa (AC).

Figure 6. Occurrence of Bothriopsis bilineatus $(\mathrm{n}=12)$ and Bothrops atrox $(\mathrm{n}=12)$ in lakes and terra firme trails at Moa river forest (AC).
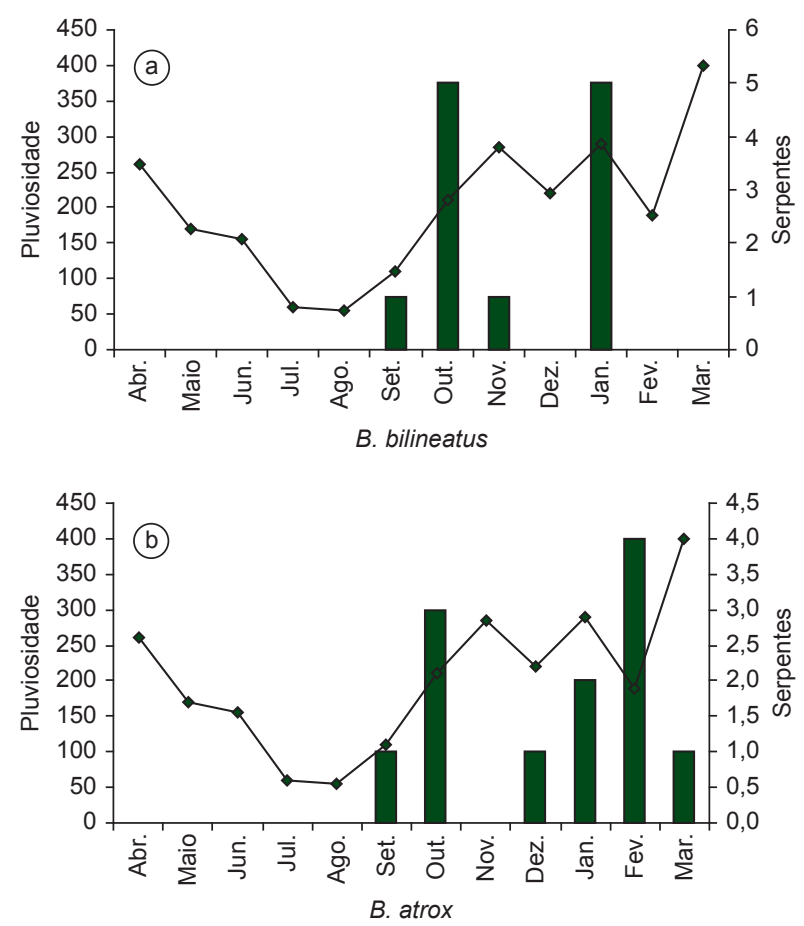

Figura 7. Ocorrência de Bothriopsis bilineatus (colunas) e de Bothrops atrox (colunas) e a pluviosidade (linhas) entre abril de 2008 a março de 2009 na floresta do Rio Moa (AC).

Figure 7. Occurrence of Bothriopsis bilineatus (bars) and of Bothrops atrox (bars) and rainfall (line) from April 2008 to March 2009 at Moa river forest (AC).

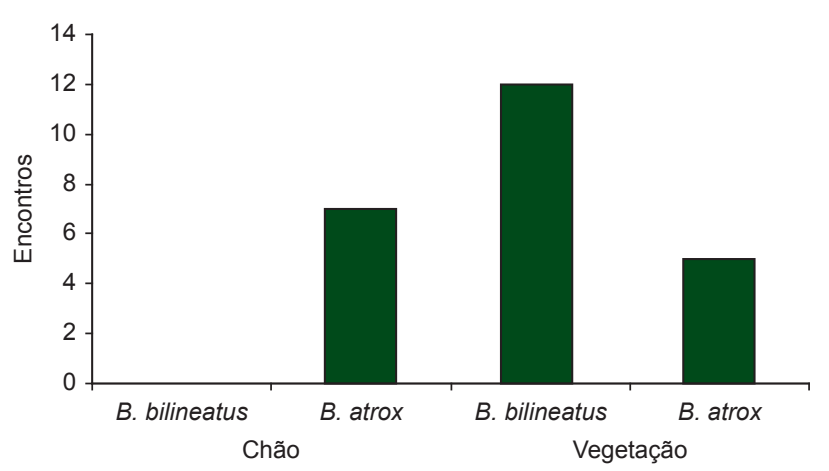

Figura 8. Ocorrência de indivíduos de Bothrops atrox $(\mathrm{n}=12)$ e de Bothriopsis bilineatus $(\mathrm{n}=12)$ sobre o chão e na vegetação na Floresta do Rio Moa (AC).

Figure 8. Occurrence of individuals of Bothrops atrox $(\mathrm{n}=12)$ and Bothriopsis bilineatus $(\mathrm{n}=12)$ on the ground and on vegetation at the Moa river forest (AC).

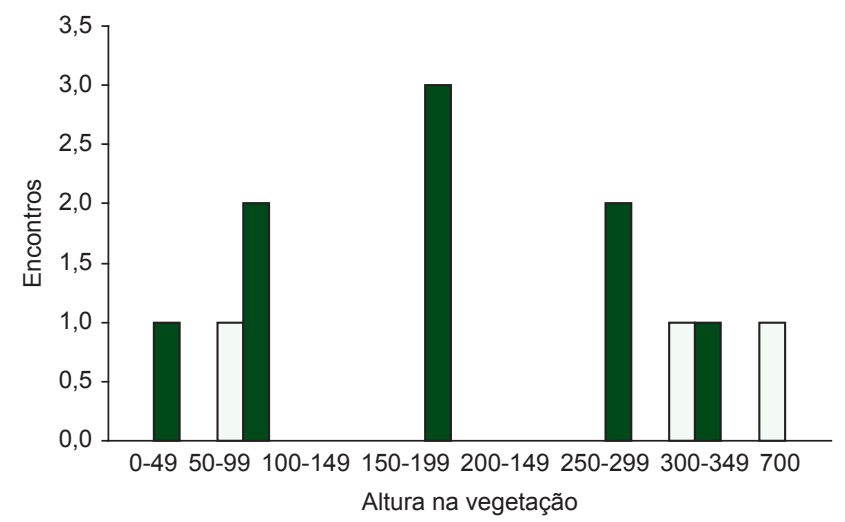

Figura 9. Ocorrência de indivíduos de Bothriopsis bilineatus $(\mathrm{n}=12)$ sobre a vegetação durante a noite (colunas verdes escuras) e de dia (colunas brancas) na Floresta do Rio Moa (AC).

Figure 9. Occurrence of individuals of Bothriopsis bilineatus $(\mathrm{n}=12)$ on vegetation at night (dark green bars) and by day (white bars) at Moa river forest $(\mathrm{AC})$.

lagos (Figura 6). Durante a PVLT, B. bilineatus foi mais frequentemente encontrada do que B. atrox (Tabela 2). Os encontros dessas serpentes ocorreram entre os meses de setembro a março (Figura 7), nenhum indivíduo foi encontrado durante os meses de abril a agosto, período que correspondeu ao período mais seco.

Todos os encontros de Bothriopsis bilineatus ocorreram sobre a vegetação (Tabela 1, Figuras 8 e 9), enquanto que de Bothrops atrox foram encontrados cinco (todos juvenis) sobre esse substrato. Os indivíduos de $B$. bilineatus foram observados em alturas de 30 a $700 \mathrm{~cm}(\overline{\mathrm{x}}=218 \mathrm{~cm})$, em atividade durante a noite (entre 30 a 
$700 \mathrm{~cm}$ ) e em repouso durante o dia (entre 50 a $300 \mathrm{~cm}$ ) (Figura 9). Aparentemente não existem diferenças na altura dos poleiros da vegetação utilizados por indivíduos juvenis e adultos e também entre a altura dos poleiros de repouso e atividade (Figuras 9 e 10). Sete indivíduos de $B$. bilineatus foram observados em atividade (seis adultos e um juvenil) (Figuras 11e 12, Tabela 2) e quatro em repouso (três adultos e dois juvenis) (Figuras 13, 14 e 15, Tabela 2). Os juvenis de B. atrox foram observados em alturas de 30 a $150 \mathrm{~cm}$ $(\overline{\mathrm{x}}=62 \mathrm{~cm})$ acima do solo, em atividade de caça por espreita ou em deslocamento, durante o período noturno. Nove indivíduos de $B$. atrox foram encontrados em atividade (três adultos e seis juvenis) (Figuras 16 e 17, Tabela 2), dois em repouso (um adulto e um juvenil) e um juvenil morto (Tabela 2).

Foram realizadas observações de interações entre três indivíduos (uma fêmea e dois machos) de Bothriopsis bilineatus. Uma fêmea (CRA $68 \mathrm{~cm}$, CC $10 \mathrm{~cm}$, P $100 \mathrm{~g}$ ) foi observada caçando de espreita na noite de 27 de janeiro de 2009 (Figura 11). Esta fêmea foi capturada para a obtenção de medidas de tamanho, solta e, após 40 minutos, retornou ao mesmo local e adotou novamente postura de caça de espreita. Na noite seguinte, às 20:20 horas, esse indivíduo foi encontrado

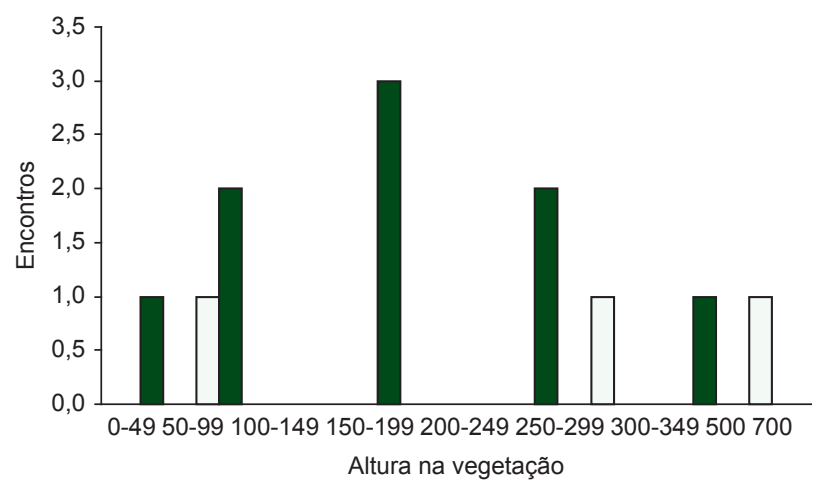

Figura 10. Ocorrência de juvenis (colunas brancas) e de adultos (colunas verde escuras) de Bothriopsis bilineatus $(\mathrm{n}=12)$ sobre a vegetação na Floresta do Rio Moa (AC).

Figure 10. Occurrence of juveniles (white bars) and adults (dark green bars) of Bothriopsis bilineatus $(\mathrm{n}=12)$ on vegetation at Moa river forest $(\mathrm{AC})$.

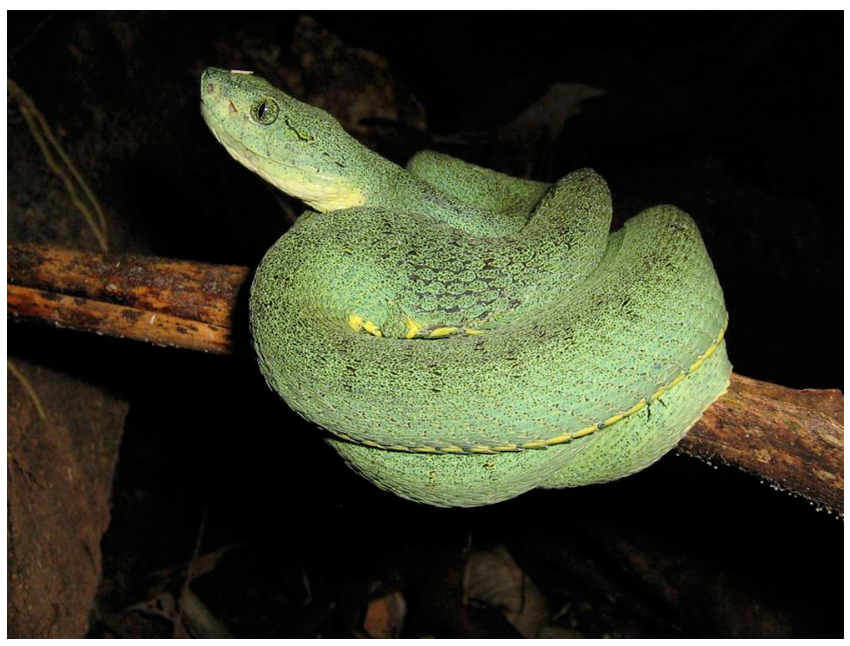

Figura 11. Fêmea adulta de Bothriopsis bilineatus em atividade sobre a vegetação durante a noite na floresta do Rio Moa (AC).

Figure 11. Active adult female of Bothriopsis bilineatus on vegetation at night at Moa river forest (AC). em repouso a aproximadamente $80 \mathrm{~cm}$ do local anterior, com volume indicando que havia ingerido alguma presa (Figura 18); ao seu lado havia um macho (CRA $46 \mathrm{~cm}, \mathrm{CC} 7 \mathrm{~cm}, \mathrm{P} 40 \mathrm{~g}$ ) e à cerca de $500 \mathrm{~cm}$ de altura outro (CRA $57 \mathrm{~cm}$, CC $10 \mathrm{~cm}, \mathrm{P} 55 \mathrm{~g}$ ). Às 20:30 horas o macho que estava na vegetação começou a se aproximar do casal, estando a uma altura de $300 \mathrm{~cm}$. Entre 20:45 e 21:22 horas o macho menor deslocou-se para cima da fêmea e permaneceu enrolado sobre ela. O macho maior, às 20:52 horas, ficou cerca de $80 \mathrm{~cm}$ do casal e permaneceu com a cabeça direcionada para eles. Às 20:54 horas este mesmo macho ficou a uma distância de $30 \mathrm{~cm}$ do casal e, às 20:57 horas, se aproximou mais ainda (Figura 19) e permaneceu com a cabeça direcionada para eles até que, às 21:22 horas, o macho menor se afastou. $\mathrm{O}$ macho maior deslocou-se para cima da fêmea (Figura 20) e começou a se mover sobre seu corpo às 21:45 horas e, às 22:15 horas, enrodilhou-se ao seu lado. Esse macho exibiu comportamento de corte, que não mudou sua posição ou reagiu as suas tentativas. A fêmea permaneceu indiferente aos movimentos dos dois machos. Às 23:00 horas esses indivíduos foram capturados para tomada das suas medidas. A fêmea coletada apresentou um roedor (P $60 \mathrm{~g}$ ) em seu trato digestivo.

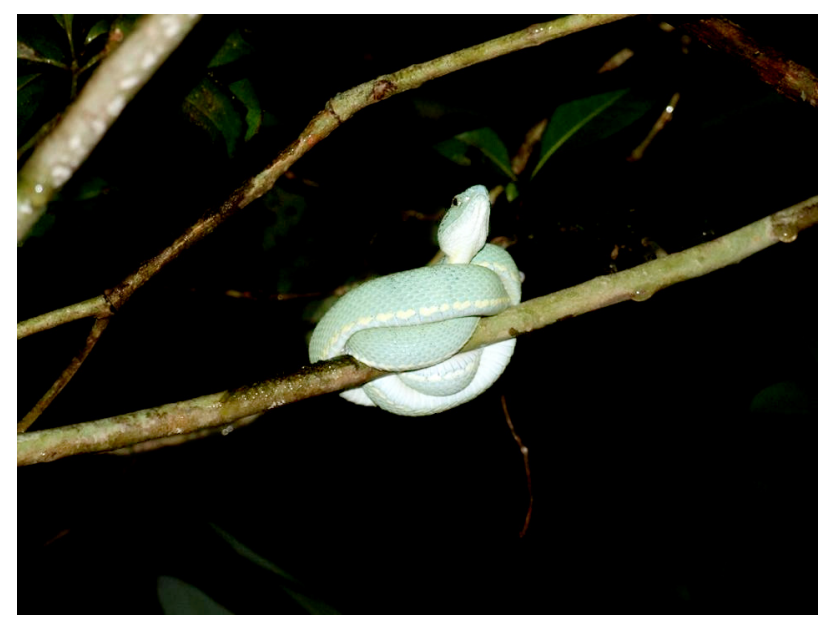

Figura 12. Adulto de Bothriopsis bilineatus em atividade sobre a vegetação durante a noite na floresta do Rio Moa (AC).

Figure 12. Active adult of Bothriopsis bilineatus on vegetation at night at Moa river forest (AC).

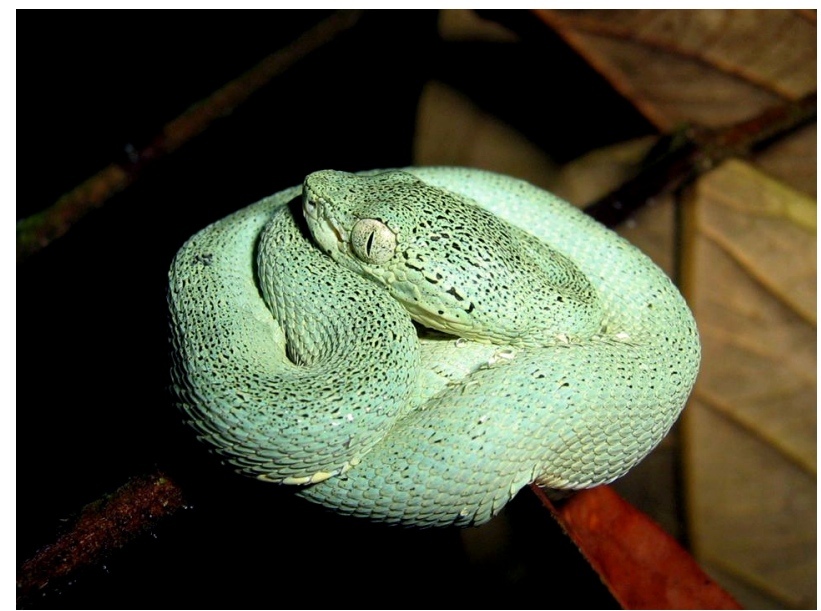

Figura 13. Juvenil de Bothriopsis bilineatus em repouso sobre a vegetação durante o dia na floresta do Rio Moa (AC).

Figure 13. Juvenile of Bothriopsis bilineatus resting on vegetation by day at Moa river forest (AC). 


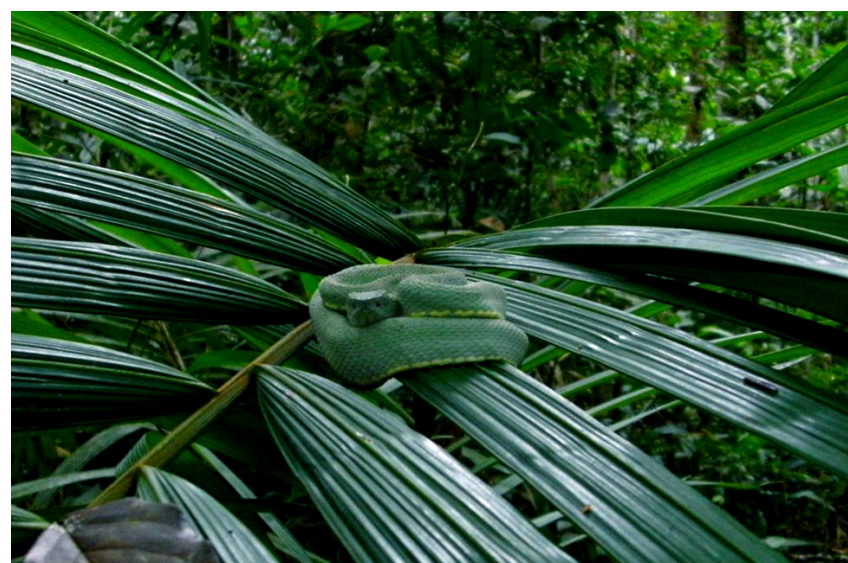

Figura 14. Adulto de Bothriopsis bilineatus em repouso sobre a vegetação durante o dia na floresta do Rio Moa (AC).

Figure 14. Adult of Bothriopsis bilineatus resting on vegetation by day at Moa river forest (AC).

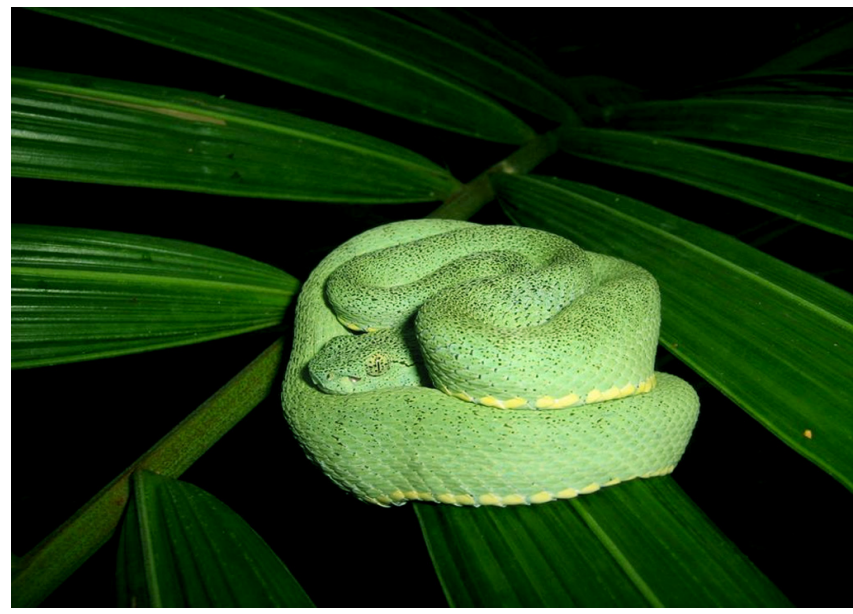

Figura 15. Mesmo indivíduo adulto de Bothriopsis bilineatus da Figura 14 em repouso sobre a vegetação durante o dia na floresta do Rio Moa (AC).

Figure 15. The same adult individual of Bothriopsis bilineatus of figure 14 resting on vegetation by day at Moa river forest (AC).

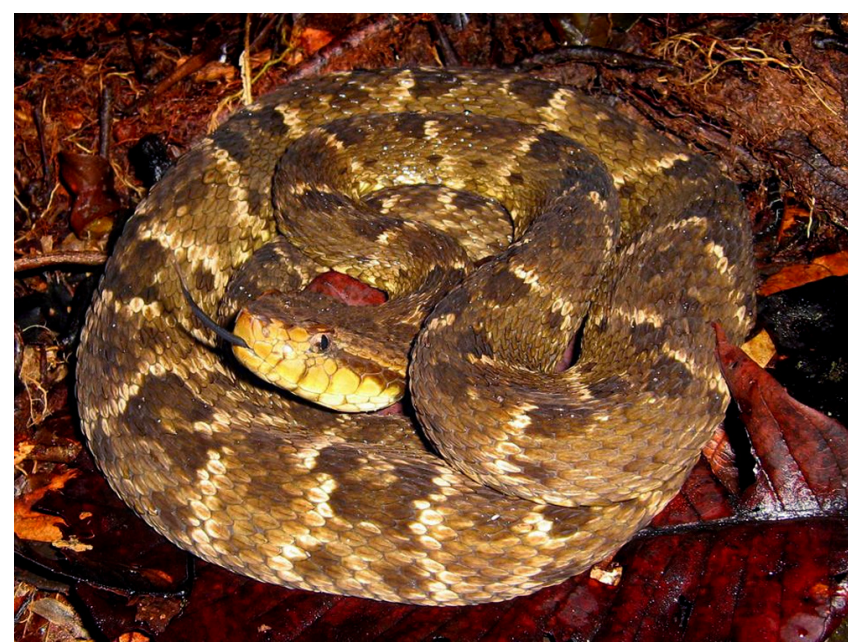

Figura 16. Adulto de Bothrops atrox em atividade no chão durante a noite na floresta do Rio Moa (AC)

Figure 16. Active adult of Bothrops atrox on the ground at night at Moa river forest $(\mathrm{AC})$.

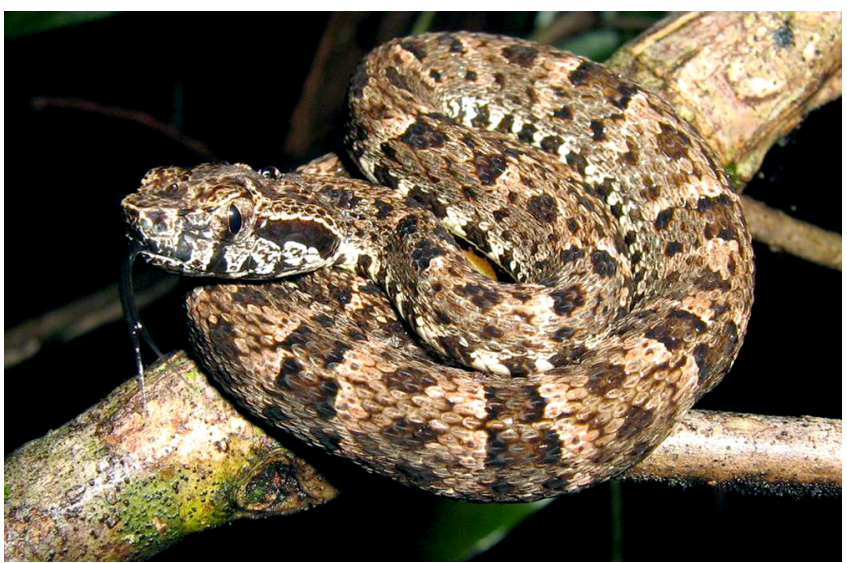

Figura 17. Juvenil de Bothrops atrox em atividade sobre a vegetação durante a noite na floresta do Rio Moa (AC).

Figure 17. Active juvenile of Bothrops atrox on vegetation at night at Moa river forest (AC).

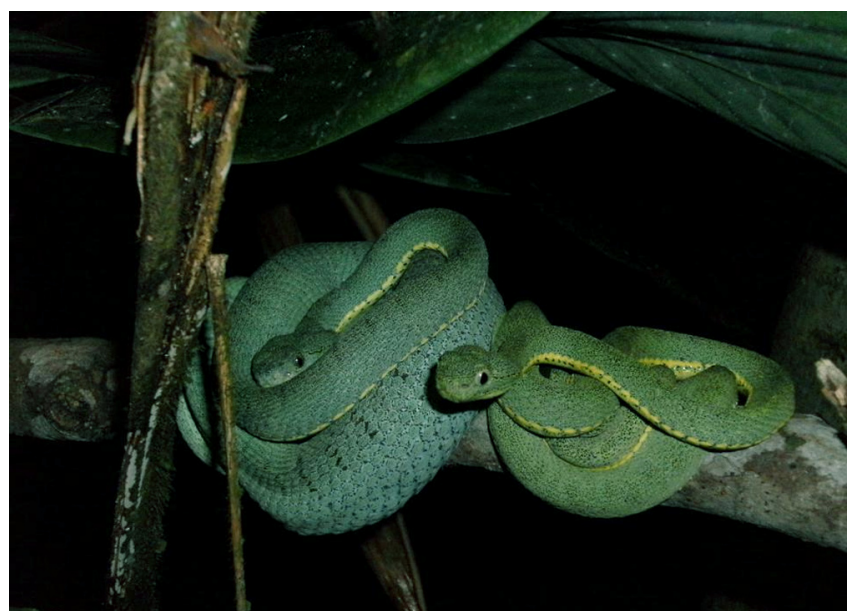

Figura 18. Fêmea e macho de Bothriopsis bilineatus sobre a vegetação durante a noite na floresta do Rio Moa (AC).

Figure 18. Female (left) and male (right) of Bothriopsis bilineatus on vegetation at night at Moa river forest (AC).

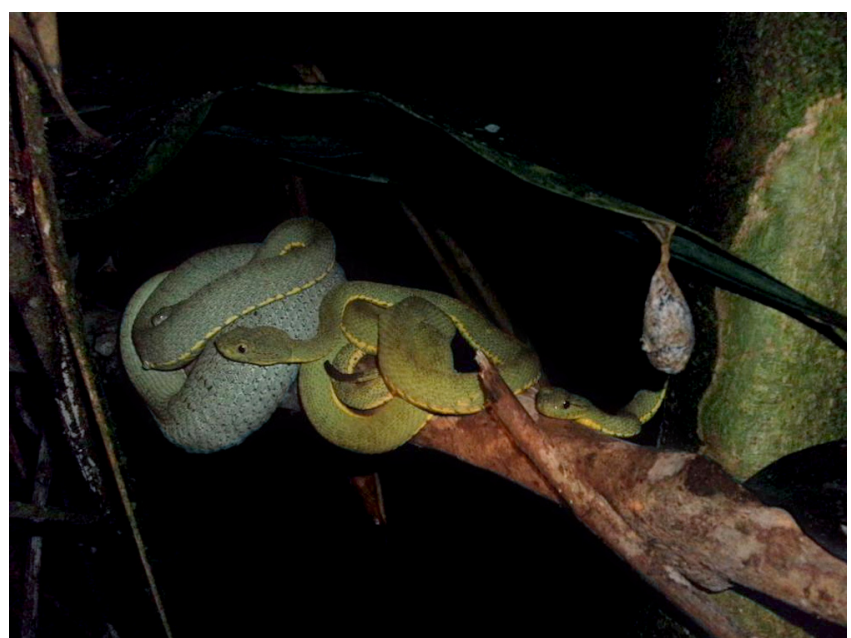

Figura 19. Chegada de um macho maior (direita) no local da fêmea (esquerda) e do macho menor (meio) de Bothriopsis bilineatus.

Figure 19. Arrival of the larger male (right) to the site where the female (left) and the small male (center) of Bothriopsis bilineatus were found. 


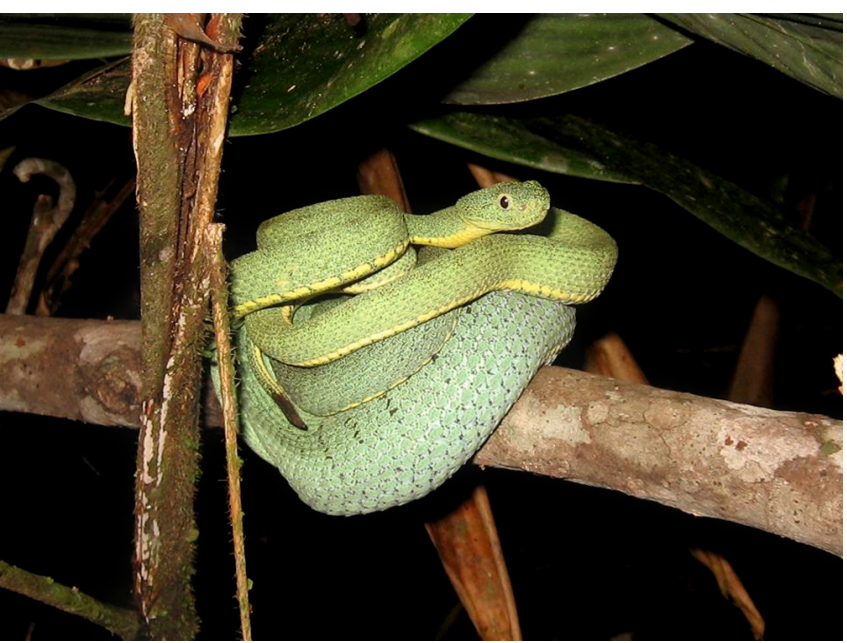

Figura 20. Macho maior sobre a fêmea de Bothriopsis bilineatus.

Figure 20. Larger male over the female Bothriopsis bilineatus.

\section{Discussão}

O hábito generalista da maioria das espécies de Bothrops (incluindo Bothriopsis) é um dos fatores responsáveis pela alta abundância dessas espécies na maioria dos habitats (Martins et al. 2002). Bothrops atrox geralmente é uma das espécies mais abundantes nos estudos realizados sobre comunidades de serpentes na Amazônia (Duellman 1978, Dixon \& Soini 1986, Cunha \& Nascimento 1993, Martins \& Oliveira 1998, Oliveira \& Martins 2001, Frota 2004, Frota et al. 2005), exceção durante o enchimento do lago da Usina Hidrelétrica de Samuel (RO) onde foram coletados 13 espécimes do total de 1016 serpentes (Jorge-da-Silva 1993) e em Espigão do Oeste (RO), onde foram registrados nove indivíduos do total de 462 (Bernarde \& Abe 2006). Durante a procura noturna nesse estudo, essa espécie teve uma taxa de encontro similar ao observado por Oliveira \& Martins (2001) para Reserva Ducke em Manaus, Amazonas (0,020 serpentes/ hora).

Diferentemente de Bothrops atrox, a espécie Bothriopsis bilineatus não costuma ser freqüente nas localidades em que ocorre (Duellman 1978, Dixon \& Soini 1986, Cunha \& Nascimento 1993, Jorge-da-Silva 1993, Frota et al. 2005, Bernarde \& Abe 2006). Cunha \& Nascimento (1993) consideraram essa espécie como rara na região Leste do Pará; no resgate de serpentes na Usina Hidrelétrica de Samuel (RO), foram obtidos quatro indivíduos (Jorge-da-Silva 1993) e em Espigão do Oeste (RO), apenas um (Bernarde \& Abe 2006). Neste estudo, B. bilineatus foi mais frequiente $(0,03$ serpente $\mathrm{p} / \mathrm{hora})$ do que B. atrox $(0,016)$ durante a PVLT.

Oliveira \& Martins (2001) observaram uma diminuição da atividade de Bothrops atrox durante a estação seca em Manaus (AM). No presente estudo não foram observados indivíduos de Bothriopsis bilineatus e B. atrox durante os meses mais secos do ano. Um dos efeitos da pluviosidade sobre a atividade das serpentes é indireto, afetando a disponibilidade de suas presas (Henderson et al. 1978, Marques et al. 2000). Por outro lado, a redução da produtividade nas florestas durante os períodos secos pode simultaneamente afetar a atividade dos animais, incluindo as serpentes e suas presas (Oliveira \& Martins 2001). Na floresta do Rio Moa ocorre uma diminuição da abundância de anuros evidenciada na menor captura destes em armadilhas de interceptação e queda nesses meses mais secos (obs. pess.), podendo isso ser um dos fatores que influenciam na atividade dessas duas serpentes nesse período
Algumas espécies de jararacas costumam ser mais freqüentes em determinados ambientes, principalmente aquáticos. Bothrops moojeni teve uma taxa de encontro por PVLT maior em ambientes ripários (0,051 serpente p/hora) do que nas áreas de interflúvio no Cerrado (Nogueira et al. 2003). Outra espécie habitante do Cerrado, B. pauloensis, ao contrário de B. moojeni, foi mais encontrada $(0,266)$ nos ambientes mais secos (Valdujo et al. 2002). Os tipos de presas utilizadas por essas espécies podem estar relacionadas a essas diferenças no uso do hábitat (Valdujo et al. 2002, Nogueira et al. 2003). Juvenis de $B$. jararaca tiveram uma alta taxa de encontro $(0,34)$ em córregos na Mata Atlântica, onde ocorre uma maior abundância de anuros, que constituem seu principal tipo de presa (Hartmann et al. 2003). A disponibilidade de presas é um dos principais fatores relacionados com o uso do hábitat pelas espécies de jararacas (Martins et al. 2001). Apesar do número de indivíduos amostrados nesse estudo ser relativamente baixo (12 encontros de cada espécie), foi observado um maior encontro de B. atrox na trilha dos lagos e de B. bilineatus na trilha de terra firme. Essas duas espécies possuem hábitos generalistas quanto a dieta (Martins \& Oliveira 1998, Martins et al. 2002) e seus tipos de presas (anuros, roedores, marsupiais) foram observados nas duas trilhas (obs. pess.). Entretanto, pelo menos no caso dos anuros, ocorrem diferenças na composição de espécies nas duas trilhas. As duas espécies diferem quanto ao uso do microhabitat (Duellman 1978, Dixon \& Soini 1986, Oliveira \& Martins 2001, Martins et al. 2001, este estudo), sendo B. atrox mais frequentemente encontrada no chão (exceção dos juvenis) e B. bilineatus principalmente arborícola. Existem diferenças na estrutura e composição da vegetação entre as duas trilhas (ver Material e Métodos) e a trilha dos lagos é alagada sazonalmente. Diferenças no hábitat (estrutura e composição da vegetação, tipos de ambientes aquáticos presentes, disponibilidades de presas) provavelmente são os fatores que determinam a ocorrência dessas duas espécies nesses locais na floresta. Além disso, essas duas espécies pertencem a diferentes linhagens dentro de Crotalinae (Fenwick et al. 2009), portanto fatores históricos possivelmente também são responsáveis por essas diferenças.

Indivíduos juvenis de Bothrops atrox foram mais encontrados sobre a vegetação do que os adultos na Amazônia central (Oliveira \& Martins 2001) e a mesma tendência foi observada neste estudo. Essa mudança ontogenética no uso do microhábitat pode estar relacionada com a disponibilidade de anuros sobre a vegetação e também pode ser uma estratégia de defesa para evitar predadores terrestres (Martins 1993, Oliveira \& Martins 2001). Quando a Bothriopsis bilineatus, nesse estudo não foram observadas diferenças nas alturas na vegetação entre juvenis e adultos, embora o número de indivíduos observados (12) tenha sido relativamente baixo para inferir padrões no uso do microhabitat nessa espécie. Na Amazônia, algumas espécies de serpentes diurnas são encontradas repousando sobre a vegetação como forma de evitar predadores terrestres (Martins 1993, Martins \& Oliveira 1998, Bernarde \& Abe 2006). Durante o dia, indivíduos adultos de B. atrox podem ser encontrados no chão (Oliveira \& Martins 2001), fato que talvez seja relacionado a defesa (espécie relativamente grande e peçonhenta, coloração críptica). Nesse estudo, os espécimes de $B$. bilineatus foram encontrados em repouso sobre a vegetação durante o dia, o que pode ser uma forma de evitar predadores terrestres (Martins 1993). Sua coloração verde deve torná-la críptica no microhábitat arbóreo, assim como ocorre em algumas espécies de colubrídeos arborícolas (Martins et al. 2008).

Eventos de comportamento de reprodução em serpentes (combate entre machos, corte, cópula) são raramente observados na natureza (Carpenter 1977, 1984), com poucos registros feitos na América do Sul (e. g. Martins \& Oliveira 1998, Feio et al. 1999, Almeida-Santos \& Marques 2002). Aparentemente os dois machos de Bothriopsis bilineatus observados exibiram comportamento de corte (ver Car- 
penter 1977), aproximando-se e tocando a fêmea. Talvez a fêmea não estivesse receptiva (e, por isso, não tenha ocorrido cópula), pelo fato de ter ingerido uma grande presa $(\mathrm{RM}=0,60)$ e por encontrar-se em repouso. $\mathrm{O}$ fato do macho menor ter se afastado com a aproximação do maior pode ser um caso de submissão e dominância (Carpenter 1984), apesar de não ter ocorrido combate entre eles.

A razão de massa observada $(0,60)$ é relativamente grande para B. bilineatus, visto que esta espécie é arborícola e possui corpo relativamente delgado. Grandes razões de massa foram observadas também para algumas espécies de Bothrops, principalmente nas de corpo mais robustos e terrícolas (Martins et al. 2002).

Em conclusão, Bothrops bilineatus e Bothrops atrox foram relativamente freqüentes nesse estudo. Geralmente B. bilineatus é pouco freqüente nos estudos herpetofaunísticos na Amazônia, mas no baixo Rio Moa ela foi abundante. Essas duas espécies apresentaram diferenças no uso do espaço, sendo que a primeira espécie apresentou hábito mais arborícola e ocorreu principalmente na área da floresta de terra firme, rica em palmeiras e que não é sazonalmente alagada. Bothrops atrox, espécie com adultos predominantemente terrícolas e juvenis com hábitos subarborícolas, foi mais abundante na área da floresta próxima aos lagos, que é sazonalmente alagada. Fatores históricos e diferenças entre essas duas áreas, como a estrutura da vegetação, tipos de ambientes aquáticos e disponibilidade de presas, são possivelmente os fatores responsáveis pela diferença na ocorrência dessas espécies. Essas duas serpentes não foram observadas nos meses mais secos, período em que também existe menor disponibilidade de anuros nesses ambientes.

\section{Agradecimentos}

A Reginaldo A. Machado pela ajuda em campo em algumas procuras. A Flávio Amorim Obermüller pela caracterização da vegetação na área de estudo. Ao CNPq pelo auxílio financeiro (Processo: 479225/2007-9). As bolsas de mestrado concedidas a Luiz Carlos B. Turci (CNPq), Saymon de Albuquerque (Capes) e Daniele B. Miranda (Capes). Ao Ibama (SISBIO) pela licença concedida ( $\mathrm{n}^{\circ}$ 12178-1 e 12178-2). A Cristiano Nogueira, Márcio Martins e um revisor anônimo da Biota Neotropica pelas sugestões.

\section{Referências Bibliográficas}

ALMEIDA-SANTOS, S.M. \& MARQUES, O.A.V. 2002. Male-Male ritual combat in the colubridae snake Chironius bicarinatus from the Atlantic Forest, southeastern Brazil. Amp. Rep. 23(4):528-533.

ÁVILA-PIRES, T.C.S., HOOGMOED, M.S. \& VITT, L.J. 2007. Herpetofauna da Amazônia. In Herpetologia no Brasil II. (L.B. Nascimento \& M.E. Oliveira, eds.). Sociedade Brasileira de Herpetologia, Belo Horizonte, p. 13-43.

BERNARDE, P.S. \& ABE, A.S. 2006. A snake community at Espigão do Oeste, Rondônia, Southwestern Amazon, Brazil. South Am. J. Herpetol. 1(2):102-113.

CAMPBELL, H.W. \& CHRISTMAN, S.P. 1982. Field techniques for herpetofaunal community analysis. In Herpetological communities: a symposium of the society for the study of amphibians and reptiles and the herpetologists' league. (N.J. Scott Jr., ed.). U.S. Fish Wildlife Service, Washington, p. 193-200.

CARPENTER, C.C. 1977. Communication and displays of snakes. Amer. Zool. 17(1):217-224

CARPENTER, C.C. 1984. Dominance in snakes. In Vertebrate ecology and systematics: a tribute to Henry S. Fitch. (R.A. Seigel, L.E. Hunt, J.L. Knight, L. Malaret \& N.L. Zuschlag, eds). The University of Kansas, Lawrence, p. 195-202.

CECHIN, S.Z. \& MARTINS, M. 2000. Eficiência de armadilhas de queda (pitfall traps) em amostragem de anfíbios e répteis no Brasil. Rev. Bras. de Zool. 17(3):729-740.
CUNHA, O.R. \& NASCIMENTO, F.P. 1993. Ofídios da Amazônia: as cobras da região Leste do Pará. Bol. Mus. Para. Emílio Goeldi., Sér. Zool. 9(1):1-191.

DIXON, J.R. \& SOINI, P. 1986. The reptiles of the upper Amazon basin, Iquitos region, Peru. 2 ed.

Milwaukee Public Museum, Milwaukee.

DUELLMAN, W.E. 1978. The biology of na equatorial herpetofauna in Amazonian Equador. Misc. Pub. Mus. Nat. Hist. Univ. Kansas. 65:1-352.

EGLER, S.G., OLIVEIRA, M.E. \& MARTINS, M. 1996. Bothrops atrox (Common lancehead). Foraging behavior and ophiophagy. Herpetol. Rev. 27(1):22-23.

FEIO, R.N., SANTOS, P.S., FERNANDES, R. \& FREITAS, T.S. 1999. Chironius flavolineatus. Courtship. Herpetol. Rev. 30(2):99-100.

FENWICK, A.M., GUTBERLET Jr., R.L., EVANS, J.A. \& PARKINSON, C.L. 2009. Morphological and molecular evidence for phylogeny and classification of South American pitvipers, genera Bothrops, Bothriopsis, and Bothrocophias (Serpentes: Viperidae). Zool. J. Linn. Soc. 156(3):617640.

FROTA, J.G. 2004. As serpentes da região de Itaituba, médio rio Tapajós, Pará, Brasil (Squamata). Com. Mus. Cienc. Tec. PUCRS. Ser. Zool. 17(1):9-19.

FROTA, J.G., SANTOS Jr., A.P., CHALKIDIS, H.M. \& GUEDES, A.G. 2005. As serpentes da região do baixo rio Amazonas, oeste do Estado do Pará, Brasil (Squamata). Biociências. 13(2):211-220.

HARTMANN, M.T., HARTMANN, P.A., CECHIN, S.Z. \& MARTINS, M. 2005. Feeding habits and habitat use in Bothrops pubescens (Viperidae, Crotalinae) from Southern Brazil. J. Herpetol. 39(4):664-667.

HARTMANN, P.A., HARTMANN, M.T. \& GIASSON, L.O.M. 2003. Uso do hábitat e alimentação em juvenis de Bothrops jararaca (Serpentes, Viperidae) na Mata Atlântica do sudeste do Brasil. Phyllomedusa. 235(2):35-41.

HENDERSON, R.W., DIXON, J.R. \& SOINI, P. 1978. On the seasonal incidence of tropical snakes. Milw. Publ. Mus. Contrib. Biol. Geol. 17(1):1-15.

JORGE da SILVA Jr., N. 1993. The snakes from Samuel hydroeletric power plant and vicinity, Rondônia, Brasil. Herpetol. Nat. Hist. 1(1):37-86.

LEHNER, P.N. 1996. Handbook of ethological methods. 2 ed. Cambridge University Press, Cambridge.

MACEDO-BERNARDE, L.C. \& BERNARDE, P.S. 2005. Bothrops atrox (Common Lancehead). Diet. Herpetol. Rev. 36(4):456.

MARQUES, O.A.V., ETEROVIC, A. \& ENDO, W. 2000. Seasonal activity of snakes in the Atlantic Forest in southeastern Brazil. Amp. Rep. 22(1):103-111.

MARTINS, M. 1993. Why do snakes sleep on the vegetation in Central Amazonia? Herpetol. Rev. 24(1):83-84.

MARTINS, M. \& GORDO, M. 1993. Bothrops atrox (Common Lancehead). Diet. Herpetol. Rev. 24:151-152.

MARTINS, M. \& OLIVEIRA, M.E. 1998. Natural history of snakes in forests of the Manaus region, Central Amazonia, Brazil. Herpetol. Nat. Hist. 6(2):78-150.

MARTINS, M., MARQUES, O.A.V. \& SAZIMA, I. 2002. Ecological and phylogenetic correlates of feeding habits in Neotropical pitvipers (Genus Bothrops). In Biology of the vipers. (G.W. Schuett, M. Höggren, M.E. Douglas \& H.W. Greene, eds.). Eagle Mountain Publishing, Eagle Mountain, Utah, p. 307-328.

MARTINS, M., MARQUES, O.A.V. \& SAZIMA, I. 2008. How to be arboreal and diurnal and still stay alive: microhabitat use, time of activity, and defense in neotropical forest snakes. South Am. J. Herpetol. 3(1):58-67.

MARTINS, M., ARAUJO, M.S., SAWAYA, R.J. \& NUNES, R. 2001. Diversity and evolution of macrohabitat use, body size and morphology in a monophyletic group of Neotropical pitvipers (Bothrops). J. Zool. 254(4):529-538. 
NASCIMENTO, P.F., BERNARDE, P.S. \& BUZZETTI, D.R.C. 2008. Bothrops atrox (Amazonian Lancehead). Diet. Herpetol. Rev. 39(3):353.

NOGUEIRA, C., SAWAYA, R.J. \& MARTINS, M. 2003. Ecology of the pitviper, Bothrops moojeni, in the Brazilian Cerrado. J. Herpetol. 37(4):653-659.

OLIVEIRA, M.E. \& MARTINS, M. 2001. When and where to find a pitviper: activity patterns and habitat use of the lancehead, Bothrops atrox, in central Amazonia, Brazil. Herpetol. Nat. Hist. 8(2):101-110.

OLIVEIRA, M.E. \& MARTINS, M. 2003. Bothrops atrox (Common Lancehead). Prey. Herpetol. Rev. 34(1):61-62.

SAZIMA, I. 1988. Um estudo de biologia comportamental da jararaca, Bothrops jararaca, com uso de marcas naturais. Mem. Inst. But. 50(3):83-99.
RIBEIRO, A.G. 1977. O clima do Estado do Acre. Boletim Geográfico. $35: 112-141$

VALDUJO, P.H., NOGUEIRA, C. \& MARTINS M. 2002. Ecology of Bothrops neuwiedi pauloensis (Serpentes: Viperidae: Crotalinae) in the brazilian Cerrado. J. Herpetol. 36(2):169-176.

Zoneamento Ecológico Econômico, ZEE. 2006. Programa Estadual de Zoneamento Ecológico Econômico do Estado do Acre Fase II: escala 1:250.000. SEMA, Rio Branco, 356 p.

Recebido em 27/04/09 Versão reformulada em 02/08/09 Publicado em 25/09/09 